\title{
Clinical Trials Design
}

National Cancer Institute

\section{Source}

National Cancer Institute. Clinical Trials Design. NCI Thesaurus. Code C15787.

The detailed planning of studies of the safety, efficacy, or optimum dosage schedule (if appropriate) of one or more diagnostic, therapeutic, or prophylactic drugs, devices, or techniques selected according to predetermined criteria of eligibility and observed for predefined evidence of favorable and unfavorable effects. 